\title{
Emergence of clonally related multidrug resistant Haemophilus influenzae with penicillin-binding protein 3-mediated resistance to extended-spectrum cephalosporins, Norway, 2006 to 2013
}

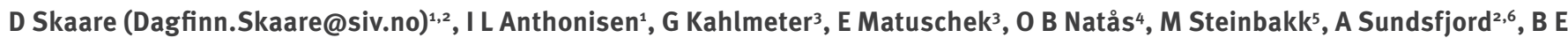
Kristiansen ${ }^{2}$

1. Department of Microbiology, Vestfold Hospital Trust, Tønsberg, Norway

2. Department of Medical Biology, Faculty of Health Sciences, University of Troms $\emptyset$ - The Arctic University of Norway, Troms $\varnothing$, Norway

3. EUCAST Development Laboratory, Växjö, Sweden

4. Department of Medical Microbiology, Stavanger University Hospital, Stavanger, Norway

5. Department of Bacteriology and Immunology, Norwegian Institute of Public Health, Oslo, Norway

6. Norwegian National Advisory Unit on Detection of Antimicrobial Resistance, University Hospital of North Norway, Troms $\emptyset$, Norway

Citation style for this article:

Citation style for this article: Haemophilus influenzae with penicillin-binding protein 3-mediated resistance to extended-spectrum cephalosporins, Norway, 2006 to 2013 . Euro Surveill. 2014;19(49): pii=20986. Available online: http://www.eurosurveillance.org/ViewArticle.aspx?Articleld=20986

Resistance to cephalosporins in Haemophilus influen$z a e$ is usually caused by characteristic alterations in penicillin-binding protein 3 ( $\left.\mathrm{PBP}_{3}\right)$, encoded by the ftsl gene. Resistance to extended-spectrum cephalosporins is associated with high-level $\mathrm{PBP}_{3}$-mediated resistance (high-rPBP3), defined by the second stage S385T substitution in addition to a first stage substitution $\left(\mathrm{R}_{517} \mathrm{H}\right.$ or $\left.\mathrm{N}_{526 \mathrm{~K}}\right)$. The third stage $\mathrm{L}_{389} \mathrm{~F}$ substitution is present in some high-rPBP3 strains. High-rPBP 3 . influenzae are considered rare outside Japan and Korea. In this study, 30 high-rPBP 3 isolates from Norway, collected between 2006 and 2013, were examined by serotyping, multilocus sequence typing (MLST), ftsl sequencing, detection of betalactamase genes and minimum inhibitory concentration (MIC) determination. MICs were interpreted according to clinical breakpoints from the European Committee on Antimicrobial Susceptibility Testing (EUCAST). Respiratory isolates predominated (proportion: $24 / 30$ ). The 30 isolates included one serotype $f$ isolate, while the remaining 29 lacked polysaccharide capsule genes. Resistance to extended-spectrum cephalosporins (cefixime, 29 isolates/30 isolates; cefepime, 28/30; cefotaxime, 26 /30; ceftaroline, $26 / 30$; ceftriaxone, $14 / 30$ ), beta-lactamase production $(11 / 30)$ and co-resistance to non-beta-lactams (trimethoprim-sulfamethoxazole, 13/30; tetracycline, 4/30; chloramphenicol, 4/30; ciprofloxacin, 3/30) was frequent. The $\mathrm{N}_{52} 6 \mathrm{~K}$ substitution in $\mathrm{PBP}_{3}$ was present in 23 of 30 isolates; these included a blood isolate which represents the first invasive $\mathrm{S}_{3} 85 \mathrm{~T}+\mathrm{N}_{5} 26 \mathrm{~K}$ isolate reported from Europe. The $\mathrm{L}_{3} 89 \mathrm{~F}$ substitution, present in 16 of 30 isolates, coincided with higher beta-lactam MICs. Non-susceptibility to meropenem was frequent in $\mathrm{S}_{3} 85 \mathrm{~T}+\mathrm{L}_{3} 89 \mathrm{~F}+\mathrm{N}_{5} 26 \mathrm{~K}$ isolates (8/12). All 11 beta-lactamase positive isolates were TEM-1. Five clonal groups of two to 10 isolates with identical MLST-ftsI allelic profiles were observed, including the first reported high-rPBP 3 clone with TEM-1 beta-lactamase and co-resistance to ciprofloxacin, tetracycline, chloramphenicol and trimethoprim-sulfamethoxazole. Prior to this study, no multidrug resistant high-rPBP3 $H$. influenzae had been reported in Norway. Intensified surveillance of antimicrobial resistance is needed to guide empiric therapy.

\section{Introduction}

Haemophilus influenzae colonises the respiratory tract in humans and infection causes a wide range of conditions, including acute otitis media, arthritis, conjunctivitis, epiglottitis, meningitis, respiratory tract infections, septicaemia and sinusitis [1]. Encapsulated isolates of serotype $b(\mathrm{Hib})$ have the highest potential for invasive disease but the introduction of Hib vaccines has dramatically reduced the burden of disease. Thus, the vast majority of infections, both invasive and non-invasive, are currently caused by nontypeable (i.e. lacking polysaccharide capsule) $H$. influenzae (NTHi) and non-b serotypes, mainly serotype $\mathrm{f}$ (Hif) [1-3].

Due to the global emergence of beta-lactamase (TEM-1 and ROB-1) producing strains in the 1970s, empiric therapy for severe disease caused by $H$. influenzae was altered from ampicillin to cephalosporins [4]. Development of resistance to extended-spectrum cephalosporins has forced a second shift in empiric treatment to carbapenems in some geographical areas [4]. The European Committee on Antimicrobial 
Susceptibility Testing (EUCAST) categorises resistance to extended-spectrum cephalosporins in $\mathrm{H}$. influenzae as an exceptional phenotype [5]. Considering such isolates 'very rare or not yet reported', EUCAST recommend that they are sent to a reference laboratory [6]. Notably, minimum inhibitory concentration (MIC) breakpoints for cephalosporins and $H$. influenzae from EUCAST are considerably lower than those from Clinical and Laboratory Standards Institute (CLSI): for instance, the susceptible minimum inhibitory concentration (MIC) breakpoint for cefotaxime from EUCAST is $\leq 0.12 \mathrm{mg} / \mathrm{L}$ while the corresponding CLSI breakpoint is $\leq 2 \mu \mathrm{g} / \mathrm{mL}[7]$.

Resistance to cephalosporins is due to alterations in the transpeptidase domain of penicillin-binding protein 3 ( $\left(\mathrm{PBP}_{3}\right.$ ), encoded by the $\mathrm{fts} / \mathrm{gene}$, with phenotypic resistance profiles depending on amino acid substitution patterns. Several genotypic classification systems for $\mathrm{PBP}_{3}$-mediated resistance ( $\mathrm{PPBP}_{3}$ ) have been suggested and the terminology is still developing $[4,8-13]$. Acquirement of one of the substitutions $\mathrm{R}_{517} \mathrm{H}$ or $\mathrm{N}_{52} 6 \mathrm{~K}$ represents the first stage of resistance development. The second stage substitution $\mathrm{S}_{3} 85 \mathrm{~T}$ is associated with increased resistance to cefotaxime and separates high-level resistant (high-rPBP3) isolates from those with low-level resistance (low-rPBP3) $[4,12,13]$. High-rPBP 3 strains may be divided into group III $\left(\mathrm{S}_{38}{ }_{5} \mathrm{~T}+\mathrm{N}_{52} 6 \mathrm{~K}\right)$ and group III-like $\left(\mathrm{S}_{3} 85 \mathrm{~T}+\mathrm{R}_{517} \mathrm{H}\right)$ [9-11]. The additional L389F substitution is associated with further increased resistance levels in high-rPBP3 strains and may be considered the third stage in development of PBP3-mediated resistance [13].

High-rPBP 3 isolates became frequent in Japan in the late 1990s [14] and in Korea during the 2000s [15]. A similar development in other geographical areas would compromise current empiric antimicrobial therapy in severe infections, which is largely based on thirdgeneration cephalosporins. However, low-rPBP3 $\mathrm{H}$. influenzae, lacking the $\mathrm{S}_{3} 85 \mathrm{~T}$ substitution and mainly susceptible to cefotaxime and ceftriaxone, still predominate in south-west and northern Europe $[2,8,11,16]$. In previous systematic surveys outside Asia where determination of $\mathrm{PBP}_{3}$ amino acid substitution patterns has been performed [2,3,8,11,16-19], very few group III isolates have been reported [1]; these include one invasive isolate from Canada [19], one otitis media isolate from France [16] and one upper respiratory tract isolate from Norway [8]. Group III-like isolates have been more frequently reported in Europe $[1,3,8,11,16,18]$.

Since 2007, the Norwegian Working Group on Antibiotics (NWGA) has recommended that cefotaxime resistant $H$. influenzae isolates are sent to the reference laboratory for $\mathrm{fts} /$ sequencing and susceptibility testing with broth microdilution. In Norway, this is carried out at Vestfold Hospital Trust, in collaboration with the Haemophilus Reference Laboratory at the Norwegian Institute of Public Health and the Norwegian National Advisory Unit on Detection of Antimicrobial Resistance at the University Hospital of North Norway. We present here susceptibility profiles, resistance genotypes and molecular strain characteristics of 30 high-rPBP3 isolates from Norway, defined by the $\mathrm{S}_{3} 85 \mathrm{~T}$ substitution, collected between May 2006 and July 2013.

\section{Methods}

\section{Isolates}

A total of 30 clinical high-rPBP3 ( $3385 \mathrm{~T}$ positive) $\mathrm{H}$. influenzae isolates from Norway, isolated at 10 different routine laboratories between May 2006 and July 2013, were included in the study. Of the 30 high-rPBP3 isolates, 27 (from 7 laboratories) with the S385T substitution were identified by partial fts/ sequencing of 39 routine isolates (from 10 laboratories) with cefotaxime $\mathrm{MIC}>0.12 \mathrm{mg} / \mathrm{L}$ (gradient test); the remaining 12 isolates were categorised as group II low-rPBP3 (N526K positive, S385T negative) and had cefotaxime $M I C=0.25 \mathrm{mg} / \mathrm{L}(\mathrm{n}=10)$ or $0.5 \mathrm{mg} / \mathrm{L}(\mathrm{n}=2)$ by gradient test. We also included three isolates (isolates 4-6) from three additional laboratories, collected in 2007 and identified as high-rPBP 3 through a previous surveillance study [8]. The 10 laboratories contributing the 30 isolates were located in mid Norway (Nord-Trøndelag county), the Oslo region (Akershus, Buskerud, Oslo and Vestfold counties), Western Norway (Sogn og Fjordane, Hordaland and Rogaland counties) and Southern Norway (Agder county).

Pure cultures were kept frozen at $-70^{\circ} \mathrm{C}$ in Microbank vials (Pro-Laboratory Diagnostics, Ontario, Canada) pending further characterisation.

\section{Demographic and clinical data}

Information about sample types, demographic data and brief clinical information (provided by the clinician at the time of sampling) were acquired from the primary laboratories. Three recently hospitalised patients with X-ray confirmed pneumonia most likely caused by study isolates were selected as case reports. For these patients, relevant supplementary information about diagnosis, antimicrobial therapy and clinical outcome was extracted from the patient files.

\section{Species identification and molecular characterisation}

Species identification was carried out by API NH (bioMérieux, Marcy-l'Etoile, France), matrix-assisted laser desorption/ionisation-time of flight (MALDI-TOF) mass spectrometry with MALDI Biotyper (Bruker Daltonics $\mathrm{GmbH}$, Bremen, Germany), and conventional methods, including absence of beta-haemolysis on blood agar. Amplification of the fucK gene when performing multilocus sequence typing (MLST) (see below) was used as an additional criterion to separate $H$. influenzae from $\mathrm{H}$. haemolyticus; all included isolates were fucK positive.

Biotypes [20] were determined using indole, urease and ornithine decarboxylase (ODC) reactions produced by API NH (bioMérieux). 
Capsular serotyping was performed at the Norwegian Institute of Public Health according to previously described methodology [21] with modifications (underscored) to the e1 (TTTGGTAACGAATGTAGTGGTAG) and e2 (ATAGCTTTACTGTATAAGTCTTAG) primers ( $5^{\prime}$ to $3^{\prime}$ ).

MLST with sequencing of the seven housekeeping genes $a d k$, atp $G, f r d B$, fuck, $m d h$, pgi and $\operatorname{rec} A$ was carried out at the Norwegian Institute of Public Health according to standard procedures [22]. Sequences were registered at http://haemophilus.mlst.net. Sequence types (ST) were assigned according to allelic profiles. STs were divided into clonal complexes (CC) (named according to founder) using eBURST Version 3 (http:// haemophilus.mlst.net/eburst).

Pulsed-field gel electrophoresis (PFGE) of selected isolates was carried out at Unilabs Telelab (Skien, Norway) as previously described [17] and clonal relatedness was interpreted according to the Tenover criteria [23].

\section{Beta-lactamase detection}

All isolates were screened for beta-lactamase production and the presence of beta-lactamase genes (TEM-1 and ROB-1) as previously described using acidimetric agar [24] and polymerase chain reaction (PCR) [17].

\section{Minimum inhibitory concentration}

\section{determination}

MICs to ampicillin, amoxicillin (ampicillin-sulbactam 2:1 ratio and amoxicillin-clavulanic acid 2:1 ratio for beta-lactamase positive isolates), cefuroxime, cefixime, cefotaxime, ceftriaxone, cefepime, imipenem, meropenem, ciprofloxacin, levofloxacin, chloramphenicol, tetracycline and trimethoprim-sulfamethoxazole were determined by broth microdilution according to CLSI methodology [25] at Vestfold Hospital Trust, using standard Sensititre plates (HPB1, STP6 and GNX3F; TREK Diagnostic Systems, Thermo Fisher Scientific, West Sussex, UK). Ceftaroline, azithromycin, clarithromycin, erythromycin, roxithromycin, telithromycin, doxycycline, minocycline and rifampicin MICs were determined at the EUCAST Development Laboratory, using custom Sensititre plates (SEFE3, TREK) with inhouse prepared EUCAST MH-F broth (cation-adjusted Mueller-Hinton broth (BBL, BD Diagnostic Systems, Franklin Lakes, NJ) with $5 \%$ lysed horse blood and $20 \mathrm{mg} / \mathrm{L}$ beta-NAD) and 16 to 20 hours incubation in sealed panels in ambient air. H. influenzae ATCC 49247 , $H$. influenzae ATCC 49766 and $H$. influenzae ATCC 35056 (ampicillin-sulbactam and amoxicillin-clavulanic acid only) were incorporated for quality control purposes, with all MICs within accepted ranges. MICs were interpreted according to EUCAST clinical breakpoints [6]. MICs of all study isolates are included in the EUCAST MIC database (www.eucast.org/MIC_distributions).
$\mathrm{ftsI} /$ penicillin-binding protein 3 typing

PCR of the fts/ gene (transpeptidase domain) was performed as described previously [17]. Sequencing was performed at GATC Biotech AG (Konstanz, Germany). Sequences were analysed with the Lasergene software (DNASTAR, Madison, WI, US). An unweighted pair group method with arithmetic mean (UPGMA) phylogram (nucleotides 1010-1719) was created using ClustalW2 (www.ebi.ac.uk) and TreeDyn (www.phylogeny.fr). $H$. influenzae Rd KW2o (GenBank accession number: CU32793) was used as reference and $H$. parainfluenzae (European Molecular Biology Laboratory (EMBL) accession number: $A B 267856)$ as outgroup. The 710 bp fragments (hereafter denoted fts/ alleles) were assigned numbers (1-10) according to frequency of occurrence and used together with MLST allelic profiles for assignment to clonal groups (CG) (see below).

Deduced $\mathrm{PBP}_{3}$ amino acid sequences (338-573) were compared with $H$. influenzae Rd $\mathrm{KW} 20$ and sequence patterns (denoted $\mathrm{PBP}_{3}$ types) were assigned numbers (1-7) according to frequency of occurrence. The substitutions $\mathrm{S}_{3} 85 \mathrm{~T}, \mathrm{R}_{517} \mathrm{H}$ and $\mathrm{N}_{52} 6 \mathrm{~K}$ were used for categorisation into $\mathrm{PBP}_{3}$ group III and group III-like [9-11]. For isolates with the third stage substitution $\mathrm{L} 389 \mathrm{~F}$, the suffix ' + ' was added. The following group designations were thus used: group III (S385T, N526K); group III+ (S385T, L389F, N526K); group III-like (S385T,

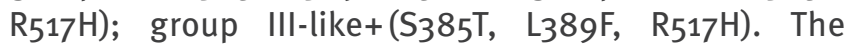
groups respectively correspond to $\mathrm{PBP}_{3}$ classes II, III, $\mathrm{V}$ and VI, proposed by Osaki et al. [13].

\section{Clonal groups}

According to a previously described approach to molecular typing of $H$. influenzae [4], CGs were defined as isolates with identical MLST-fts/ allelic profiles and numbered chronologically [1-5].

\section{GyrA and ParC amino acid substitutions}

For isolates with ciprofloxacin MIC>0.06 mg/L and/or levofloxacin MIC>0.03 mg/L, the quinolone-resistance determining regions (QRDR) of gyrA and parC were characterised by PCR methodology adapted from a previous report [26]. DNA sequencing of amplified products was carried out at GATC Biotech. Sequences were analysed with Lasergene. Deduced amino acid sequences of GyrA (64-177) and ParC (66-236) were compared with $H$. influenzae Rd KW20 (GenBank accession number: U32793).

\section{Nucleotide sequences}

The ftsl (HG983286-HG983315), gyrA (HG983316HG983320) and parC (HG983321-HG983325) sequences from this study are available at the European Nucleotide Archive (www.ebi.ac.uk).

\section{Ethics}

The use of clinical data in this study is approved by the Regional Committees for Medical and Health Research Ethics in Norway (reference number 2014/411). 
TABLE 1

Characterisation of Haemophilus influenzae with high-level penicillin-binding protein 3-mediated beta-lactam resistance (high-rPBP3), Norway, 2006-2013 (n=30)

\begin{tabular}{|c|c|c|c|c|c|c|c|c|c|c|c|c|c|c|}
\hline \multirow{3}{*}{$\begin{array}{l}\text { Isolate } \\
1 \\
\end{array}$} & \multirow{2}{*}{\multicolumn{2}{|c|}{$\begin{array}{l}\text { Year/ } \\
\text { region }\end{array}$}} & \multirow{2}{*}{\multicolumn{2}{|c|}{$\begin{array}{l}\text { Category } / \\
\text { age group }^{d}\end{array}$}} & \multirow{3}{*}{$\begin{array}{l}\text { Site }^{e} \\
\text { Nose } \\
\end{array}$} & \multirow{3}{*}{$\begin{array}{c}\text { Clinical data }^{f} \\
\text { ALL/AOM }\end{array}$} & \multirow{3}{*}{$\begin{array}{c}\text { Biotype }^{g} \\
\text { III }\end{array}$} & \multicolumn{2}{|c|}{ MLST } & \multirow{3}{*}{$\begin{array}{c}f t s /^{\mathrm{h}} \\
3 \\
\end{array}$} & \multirow{3}{*}{\begin{tabular}{c|}
$\begin{array}{c}\text { Clonal } \\
\text { group }\end{array}$ \\
1 \\
\end{tabular}} & \multicolumn{2}{|c|}{$\mathrm{PBP}_{3}$} & \multirow{3}{*}{$\begin{array}{c}\text { Co-resistance } \\
- \\
\end{array}$} \\
\hline & & & & & & & & \multirow{2}{*}{$\begin{array}{c}\text { ST } \\
155 \\
\end{array}$} & \multirow{2}{*}{$\begin{array}{l}\mathrm{CC} \\
155 \\
\end{array}$} & & & \multirow{2}{*}{\begin{tabular}{|c|} 
Type \\
4 \\
\end{tabular}} & \multirow{2}{*}{$\begin{array}{l}\text { Groupk } \\
\text { III-like }\end{array}$} & \\
\hline & 2006 & 0 & Hosp & 1 & & & & & & & & & & \\
\hline 2 & 2007 & 0 & GP & 1 & Nose & URTI & IV & 1197 & 395 & 1 & 2 & 1 & III & $\beta$ \\
\hline 3 & 2007 & 0 & GP & 5 & $\mathrm{Npx}$ & No data & III & 159 & 503 & 6 & Single & 5 & $\mathrm{III+}$ & Ts \\
\hline 4 & 2007 & 0 & $O C$ & 3 & Nose & No data & IV & 1197 & 395 & 1 & 2 & 1 & III & - \\
\hline 5 & 2007 & 0 & $O C$ & 1 & $\mathrm{Npx}$ & No data & III & 155 & 155 & 3 & 1 & 4 & III-like & - \\
\hline 6 & 2007 & $M$ & Hosp & 7 & LRT & No data & III & 155 & 155 & 3 & 1 & 4 & III-like & - \\
\hline 7 & 2008 & 0 & GP & 3 & $\mathrm{Npx}$ & No data & III & 1197 & 395 & 1 & 2 & 1 & III & - \\
\hline 8 & 2008 & 0 & Hosp & 1 & Nose & URTI & IV & 1197 & 395 & 1 & 2 & 1 & III & - \\
\hline 9 & 2008 & 0 & GP & 1 & $\mathrm{Npx}$ & No data & IV & 1197 & 395 & 1 & 2 & 1 & III & - \\
\hline 10 & 2008 & 0 & GP & 1 & Nose & No data & IV & 1197 & 395 & 1 & 2 & 1 & III & - \\
\hline 11 & 2009 & 0 & GP & 5 & Npx & Sinusitis & 1 & 408 & 3 & 7 & Single & 6 & $\mathrm{III+}$ & - \\
\hline 12 & 2009 & W & $O C$ & 1 & Ear & $\mathrm{AOM}$ & IV & 1197 & 395 & 1 & 2 & 1 & III & - \\
\hline 13 & 2009 & W & GP & 6 & Nose & Sinusitis & III & 1197 & 395 & 1 & 2 & 1 & III & - \\
\hline 14 & 2009 & W & GP & 1 & Ear & $\mathrm{AOM}$ & III & 1197 & 395 & 1 & 2 & 1 & III & - \\
\hline 15 & 2010 & W & GP & 1 & $\mathrm{Npx}$ & $\mathrm{AOM}$ & III & 1197 & 395 & 1 & 2 & 1 & III & - \\
\hline 16 & 2011 & 0 & GP & 1 & Eye & Conjunctivitis & II & 422 & 422 & 4 & 3 & 3 & III-like+ & $\beta, q$, Ts \\
\hline 17 & 2011 & 0 & GP & 4 & Nose & Sinusitis & II & 142 & 142 & 8 & Single & 3 & III-like+ & - \\
\hline 18 & 2012 & $\mathrm{~S}$ & Hosp & 8 & Blood & MM/pneumonia & 1 & 1287 & None & 9 & Single & 7 & III & - \\
\hline 19 & 2012 & W & $O C$ & 8 & BAL & COPD/DC & II & 160 & 160 & 2 & Single & 2 & III+ & Ts \\
\hline 20 & 2012 & W & $\mathrm{OC}$ & 1 & Sputum & $\mathrm{CF}$ & III & 148 & 245 & 10 & Single & 2 & $\mathrm{III+}$ & - \\
\hline 21 & 2012 & 0 & GP & 1 & Ear & $\mathrm{AOM}$ & 1 & 124 & 124 & 2 & Single & 2 & $\mathrm{III+}$ & Ts \\
\hline 22 & 2013 & W & GP & 8 & Sputum & Pneumonia & IV & 1282 & 503 & 4 & Single & 3 & III-like+ & $\beta, C, T, T s$ \\
\hline 23 & 2013 & $\mathrm{O}^{\mathrm{m}}$ & Hosp & 7 & Nose & Pneumonia & II & 422 & 422 & 4 & 3 & 3 & III-like+ & $\beta, q$, Ts \\
\hline 24 & 2013 & W & Hosp & 8 & Eye & Dacryocystitis & III & 159 & 503 & 5 & 4 & 2 & $\mathrm{III+}$ & $\beta, Q, C, T, T s$ \\
\hline 25 & 2013 & W & $\mathrm{OC}$ & 1 & $\mathrm{Npx}$ & PCD & III & 159 & 503 & 5 & 4 & 2 & $\mathrm{III+}$ & $\beta, Q, C, T, T s$ \\
\hline 26 & 2013 & W & $\mathrm{OC}$ & 4 & Sputum & CF/pharyngitis & III & 159 & 503 & 5 & 4 & 2 & $\mathrm{III+}$ & $\beta, Q, C, T, T s$ \\
\hline 27 & 2013 & W & Hosp & 8 & Sputum & CVD/pneumonia & IV & 836 & 245 & 2 & 5 & 2 & $\mathrm{III+}$ & $\beta$, Ts \\
\hline 28 & 2013 & W & Hosp & 8 & Sputum & DC/pneumonia & III & 836 & 245 & 2 & 5 & 2 & $\mathrm{III+}$ & $\beta$, Ts \\
\hline 29 & 2013 & W & Hosp & 7 & Sputum & COPD/pneumonia & IV & 836 & 245 & 2 & 5 & 2 & $\mathrm{III+}$ & $\beta, \mathrm{Ts}$ \\
\hline 30 & 2013 & W & Hosp & 8 & Npx & $\begin{array}{c}\text { Possible } \\
\text { pneumonia }\end{array}$ & III & 836 & 245 & 2 & 5 & 2 & $\mathrm{III+}$ & $\beta$, Ts \\
\hline
\end{tabular}

CC: clonal complex; MLST: multilocus sequence typing; PBP3: penicillin-binding protein 3; ST: sequence type.

a All isolates lacked polysaccharide capsule genes except for isolate 21 (serotype f).

b M: mid Norway (Nord-Trøndelag); O: Oslo region (Oslo, Akershus, Buskerud, Vestfold); S: Southern Norway (Agder); W: Western Norway (Sogn og Fjordane, Hordaland, Rogaland).

GP: general practice; Hosp: hospitalised; OC: outpatient clinic.

1: $0-9$ years; $2:$ 10-19 years; 3: 20-29 years; 4: 30-39 years; 5: 40-49 years; 6: 50-59 years; 7: 60-69 years; 8: $\geq 70$ years

BAL: bronchioalveolar lavage; LRT: lower respiratory tract; Npx: nasopharynx.

ALL: acute lymphoblastic leukemia; AOM: acute otitis media; CF: cystic fibrosis; COPD: chronic obstructive pulmonary disease; CVD: cardiovascular disease; DC: disseminated cancer; MM: multimorbidity (hypogammaglobulinemia, chronic lymphocytic leukaemia, myasthenia gravis, COPD); PCD: primary ciliary dyskinesia; URTI: upper respiratory tract infection.

g According to reactions (positive (+) or negative $(-)$ ) to indole, urease and ornithine decarboxylase (ODC) respectively. I: +/+/+; II: +/+/-; III: $-/+/-;$ IV: $-/+/+[18]$.

ftsl alleles (Figure 1).

Isolates with identical MLST-fts/ allelic profiles (Figure 1). Isolates qualified as 'single' are those among the 30 analysed with a unique MLST-fts/ allele combination.

PBP3 type according to amino acid sequences (substitutions underscored) in positions 350, 357, 377, 385, 389, 502, 517, 526, 532, 547, 557,

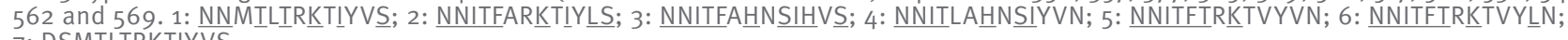
7: DSMTLIR $\underline{\text { TIIY } \underline{\mathrm{S}} \text {. }}$.

Group III: S385T and N526K; group III+: S385T, L389F and N526K; group III-like: S385T and R517H; group III-like+: S385T, L389F and R517H. B: TEM-1 beta-lactamase; Q: quinolones; q: quinolones (low level resistant); C: chloramphenicol; T: tetracyclines; Ts: trimethoprimsulfamethoxazole; -: none.

m Recently travelled to Thailand. 
Phylogram of ftsI DNA sequences from Haemophilus influenzae with high-level penicillin-binding protein 3-mediated betalactam resistance (high-rPBP3), Norway, 2006-2013 ( $\mathrm{n}=30)$

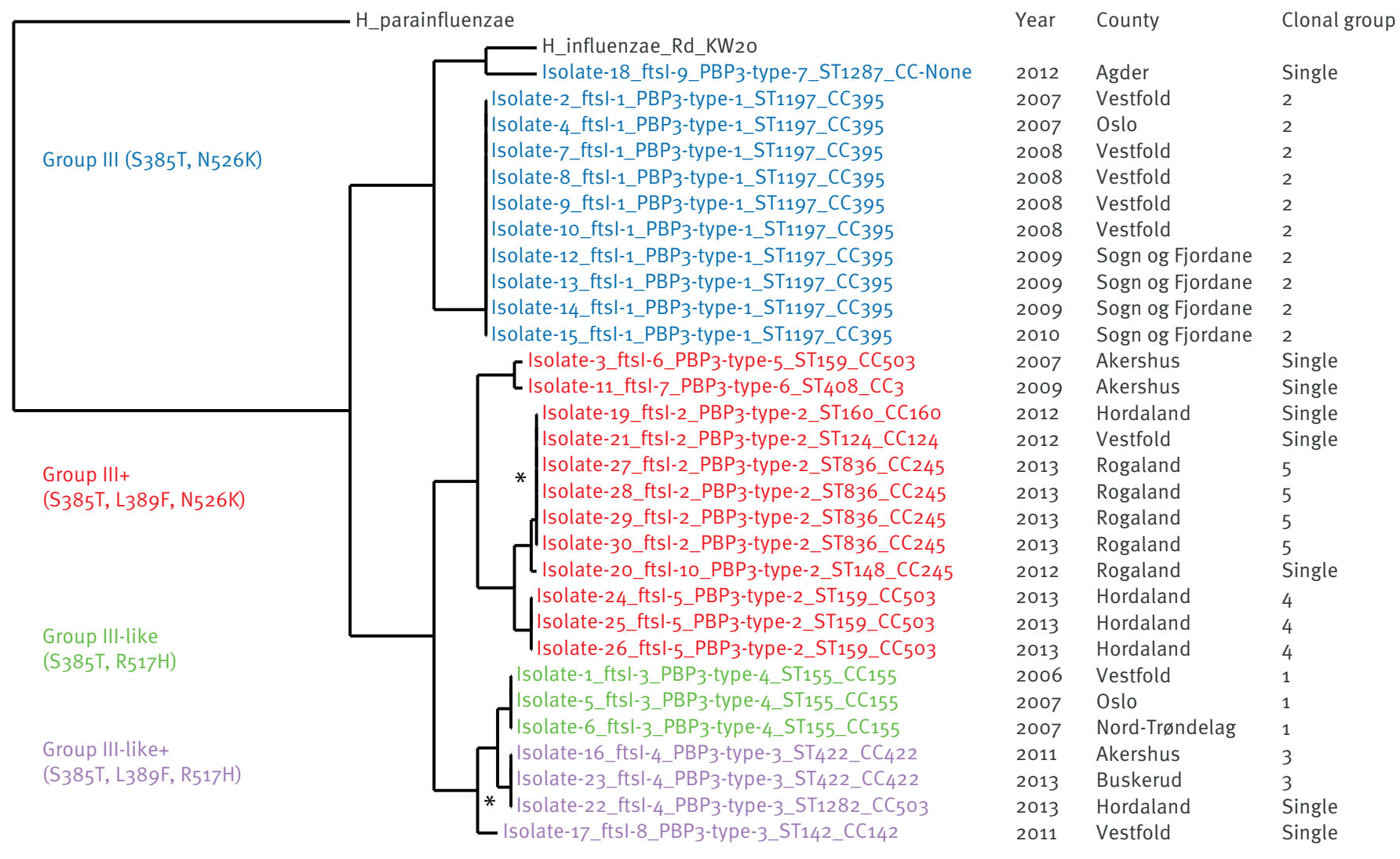

0.04

The phylogram was constructed using unweighted pair group method with arithmetic mean (UPGMA) and based on fts/ DNA sequences

(transpeptidase domain, nucleotides 1010-1719) including $H$. influenzae Rd KW20 (GenBank accession number: U32793) as reference and $H$. parainfluenzae (European Molecular Biology Laboratory (EMBL) accession number: AB267856) as outgroup.

The scale is DNA divergence.

Labels indicate isolate numbers, fts/ alleles, penicillin-binding protein 3 (РВР3) types (Table 1 ), multilocus sequence typing sequence types

(ST) and clonal complexes (CC).

Colours indicate $\mathrm{PBP}_{3}$ groups. Asterisks indicate $\mathrm{fts} /$ alleles carried by unrelated STs.

Year/county of isolation and assignment to clonal groups (CG) to the right.

\section{Results}

\section{Patient data}

The median age of the 30 patients from whom the respective isolates were derived, was 29 years (range: 0-91). In the age group zero to nine years, most patients (proportion: 10/13) were below five years-old. The male/female ratio was $13 / 17$. All but two patients lived in the Oslo region (14/30) or in Western Norway (14/30). Except for the patient with isolate 23, recently returning from Thailand, there was no known recent $(<3$ months) travel history abroad (Table 1).

Ten isolates (10/30) were from hospitalised patients. The proportion of hospitalised patients was 4/21 in the period between 2006 and 2012 compared to 6/9 in 2013. One of the 30 isolates was from blood; the rest were eye (2/30), ear $(3 / 30)$ or respiratory isolates (24/30). Among the patients for whom clinical information was available $(n=23)$, pneumonia $(n=7)$ and acute otitis media (AOM) $(n=5)$ were the most frequent infection types. At least seven of the 30 patients were predisposed for respiratory tract infections due to underlying chronic condition (Table 1).

\section{Strain characterisation}

All isolates but one (Hif) lacked polysaccharide capsule genes and were categorised as nontypeable (NTHi). The isolates were biotypes I (proportion: 3/30), II (4/30), III 
Pulsed-field gel electrophoresis (PFGE) band patterns and pulsotypes for Haemophilus influenzae isolates of clonal group 2, Norway, 2006-2013 (n=7)

\begin{tabular}{lllll} 
Isolate & Year & County & Region & Pulsotype \\
\hline Std & & & & \\
\hline 2 & 2007 & Ve & O & 3 \\
\hline 15 & 2010 & SF & W & 1 \\
\hline 13 & 2009 & SF & W & 2 \\
\hline Std & & & & \\
\hline 12 & 2009 & SF & W & 2 \\
\hline 14 & 2009 & SF & W & 1 \\
\hline 8 & 2008 & Ve & O & 1 \\
\hline 4 & 2007 & Os & 0 & 4 \\
\hline Std & & & &
\end{tabular}

O: Oslo region; Os: Oslo county; SF: Sogn og Fjordane county; Std: size standard (Staphylococcus aureus NCTC 8325); Ve: Vestfold county; W: Western Norway.

Labels indicate isolate numbers, year, county and region of isolation, and pulsotype.

The seven isolates depicted are described in Table 1. Isolate 2 is TEM-1 beta-lactamase positive, the remaining isolates are beta-lactamase negative.

$(14 / 30)$ and IV (9/30). The Hif isolate and the blood isolate were both biotype I.

Twelve STs were represented, including ST1282 and ST1287 not previously registered in the MLST database (Table 1). Eleven of the 12 STs belonged to nine CCs; the last (ST1287) could not be assigned to a CC. ST1197 (CC395) was the most frequent ST (10/30). Two STs (ST1197 and ST836) encompassed both biotype III (ODC negative) and IV isolates (ODC positive).

\section{ftsI/PBP3 genotypes}

Ten fts/ alleles, encoding seven $\mathrm{PBP}_{3}$ substitution types and carried by one to 10 isolates, were observed (Table 1). Two $\mathrm{fts} /$ alleles ( $f t s /-2$ and $f t s /-4$ ) were present in unrelated STs (Figure 1).

The majority of isolates (proportion: 23/30) had the N526K first stage substitution and were thus categorised as group III (11/30) or group III+(12/30). Seven isolates (7/30) had the $\mathrm{R}_{517} \mathrm{H}$ first stage substitution and were thus categorised as group III-like (3/30) or group III-like+(4/30).

The third stage substitution $\mathrm{L}_{3} 89 \mathrm{~F}$ was present in 16 of 30 isolates (Table 1).

\section{Clonal groups}

Five clusters comprising two to 10 isolates with identical MLST-fts/ allelic profiles (clonal groups, CG) were identified, accounting for 22 of 30 isolates. Eight isolates of 30 had unique MLST-fts/ allelic profiles, including the single Hif isolate and the single invasive isolate (Table 1).

$\mathrm{CG}_{2}(\mathrm{n}=10$ isolates) was found in two separate geographical regions (Oslo region and Western Norway) during a period of three years. To confirm genetic relationship, PFGE of seven isolates belonging to $\mathrm{CG}_{2}$ (one beta-lactamase positive and six beta-lactamase negative isolates) was performed, and four related pulsotypes (1-4) were observed (Figure 2). Pulsotypes $2(n=2), 3(n=1)$ and $4(n=1)$ differed from pulsotype $1(n=3)$ by two, two and three bands, respectively. Pulsotype 1 was observed in both geographical regions. No pulsotypes differed by more than five bands.

The three $\mathrm{CG}_{4}$ isolates were sampled during a period of four days from one hospitalised patient and two outpatients related to the same hospital. $\mathrm{CG}_{5}(\mathrm{n}=4)$ included two patients from the same household (patients 27 and 28) and was restricted to one hospital department within a period of 16 days.

The clonal groups (and the single strains) differed considerably with respect to $\mathrm{PBP}_{3}$ resistance genotypes and co-resistance (Table 1). Notably, in contrast to $\mathrm{CG}_{1}$ and $\mathrm{CG}_{2}$, the third stage $\mathrm{L}_{3} 89 \mathrm{~F}$ substitution was present in $\mathrm{CG}_{3}, \mathrm{CG}_{4}$ and $\mathrm{CG}_{5}$. $\mathrm{CG}_{1}$ and $\mathrm{CG}_{2}$ had no coresistance to non-beta-lactams, whereas $\mathrm{CG}_{4}$ was multidrug resistant. 
TABLE 2

Antimicrobial susceptibility of Haemophilus influenzae isolates with high-level penicillin-binding protein 3-mediated betalactam resistance (high-rPBP3), Norway, 2006-2013 $(\mathrm{n}=30)$

\begin{tabular}{|c|c|c|c|c|c|}
\hline Agents & MIC range (mg/L) & $\mathrm{MIC}_{50}(\mathrm{mg} / \mathrm{L})$ & MIC9o (mg/L) & $\begin{array}{c}\text { Breakpoints } \\
(\mathrm{S} \leq / \mathrm{R}>)^{\mathrm{a}}\end{array}$ & $\begin{array}{l}\mathrm{R} \text { or } \mathrm{I} / \mathrm{R} \\
\mathrm{n}(\%)^{\mathrm{b}}\end{array}$ \\
\hline Ampicillin $^{c}$ & $1-\geq 8$ & 4 & $\geq 8$ & $1 / 1$ & $29(97)$ \\
\hline Amoxicillin $^{d}$ & $4-32$ & 8 & 8 & $2 / 2$ & $30(100)$ \\
\hline Cefuroxime & $2-\geq 16$ & 8 & $\geq 16$ & $1 / 2$ & $1(3) / 29$ (97) \\
\hline Cefotaxime & $\leq 0.12-\geq 4$ & 0.25 & 1 & $0.12 / 0.12$ & $26(87)$ \\
\hline Ceftriaxone & $\leq 0.03-2$ & 0.12 & 0.5 & $0.12 / 0.12$ & $14(47)$ \\
\hline Cefixime & $0.12-\geq 2$ & 0.5 & $\geq 2$ & $0.12 / 0.12$ & $29(97)$ \\
\hline Cefepime & $0.25-\geq 4$ & 1 & 2 & $0.25 / 0.25$ & $28(93)$ \\
\hline Ceftaroline & $0.03-0.25$ & 0.12 & 0.25 & $0.03 / 0.03$ & $26(87)$ \\
\hline Imipenem & $\leq 0.5-2$ & 1 & 1 & $2 / 2$ & o (o) \\
\hline Meropenem $^{e}$ & $0.12-2$ & 0.25 & 0.5 & $0.25 / 1^{\mathrm{e}}$ & $9(30) / 1(3)$ \\
\hline Ciprofloxacin & $\leq 0.06-2$ & $\leq 0.06$ & 0.25 & $0.5 / 0.5$ & $3(10)$ \\
\hline Levofloxacin & $\leq 0.03-1$ & $\leq 0.03$ & 0.25 & $1 / 1$ & $\mathrm{o}(\mathrm{o})$ \\
\hline Chloramphenicol & $0.25-\geq 8$ & 0.5 & $\geq 8$ & $2 / 2$ & $4(13)$ \\
\hline Rifampicin $^{f}$ & $0.25-1$ & 0.5 & 0.5 & $1 / 1^{f}$ & $\mathrm{o}(\mathrm{o})$ \\
\hline Tetracycline & $0.5-\geq 8$ & 0.5 & $\geq 8$ & $1 / 2$ & o (o) / 4 (13) \\
\hline Doxycycline & $0.25-2$ & 0.25 & 2 & $1 / 2$ & $4(13) / 0(0)$ \\
\hline Minocycline & $0.12-0.5$ & 0.25 & 0.5 & $1 / 2$ & $\mathrm{o}(\mathrm{o}) / \mathrm{o}(\mathrm{o})$ \\
\hline Trimethoprim-sulfamethoxazole & $0.12-\geq 4$ & 0.5 & $\geq 4$ & $0.5 / 1$ & $0(0) / 13(43)$ \\
\hline Erythromycing & $2-4$ & 2 & 4 & $0.5 / 16^{\mathrm{g}}$ & $30(100) / 0(0)$ \\
\hline Clarithromycing & $2-16$ & 4 & 8 & $1 / 32^{g}$ & $30(100) / 0(0)$ \\
\hline Azithromycing & $\leq 0.25-1$ & 0.5 & 0.5 & $0.12 / 4^{g}$ & $30(100) / 0(0)$ \\
\hline Roxithromycing & $2-16$ & 4 & 8 & $1 / 16^{g}$ & $30(100) / 0(0)$ \\
\hline Telithromycins & $0.5-2$ & 1 & 1 & $0.12 / 8^{g}$ & $30(100) / 0(0)$ \\
\hline
\end{tabular}

I: intermediate; MIC: minimum inhibitory concentration; R: resistant; S: susceptible.

European Committee on Antimicrobial Susceptibility Testing (EUCAST) clinical breakpoints [6]. The I category is inferred from the S and R breakpoints.

b Isolates categorised as R (when only S and R categories exist) or as I/R (when an I category is defined).

Beta-lactamase positive isolates $(n=11)$ tested in the presence of sulbactam (2:1 ratio).

Beta-lactamase positive isolates $(n=11)$ tested in the presence of clavulanic acid ( $2: 1$ ratio).

Meningitis breakpoints. All isolates were susceptible according to general breakpoints $(2 / 2)$.

Prophylaxis breakpoints.

g Macrolide breakpoints categorise the wild type population as intermediately susceptible.

\section{Penicillin-binding protein 3-mediated resistance}

All strains were categorised as resistant to at least one of the extended-spectrum cephalosporins cefotaxime, ceftriaxone, cefixime, cefepime and ceftaroline; proportions of resistant isolates varied from $14 / 30$ for ceftriaxone to $29 / 30$ for the oral agent cefixime (Table 2 ). In general, strains with the $\mathrm{N}_{52} 2 \mathrm{~K}$ first stage substitution had higher MICs to extended-spectrum cephalosporins (except cefixime) than $\mathrm{R}_{517} \mathrm{H}$ strains. Furthermore, the third stage $\mathrm{L}_{389} \mathrm{~F}$ substitution was generally associated with higher MICs to extended-spectrum cephalosporins and meropenem. Notably, eight of 12 group $\mathrm{III+}$ isolates ( $\mathrm{N}_{526} \mathrm{~K}, \mathrm{~S}_{3} 85^{\mathrm{T}}$ and $\mathrm{L}_{3} 89 \mathrm{~F}$ ) were non-susceptible to meropenem (Table 3 ).
Individual susceptibility profiles and resistance genotypes are shown in Figure 3. In contrast to other group III isolates, the single invasive ST1287 isolate (isolate number 18 ) was categorised as susceptible to ampicillin $(M I C=1 \mathrm{mg} / \mathrm{L})$, cefotaxime $(M I C \leq 0.12 \mathrm{mg} / \mathrm{L})$, ceftriaxone $(\mathrm{MIC} \leq 0.03 \mathrm{mg} / \mathrm{L})$, cefixime $(\mathrm{MIC}=0.12 \mathrm{mg} / \mathrm{L})$ and cefepime $(M I C=0.25 \mathrm{mg} / \mathrm{L})$. Conversely, the single ST124 Hif isolate (isolate number 21) generally expressed higher resistance levels to beta-lactams than other group III+ isolates.

Figure 3. Year-to-year overview of Haemophilus influenzae isolates with high-level penicillin-binding protein 3-mediated beta-lactam resistance (high-rPBP3), Norway, 2006-2013 $(n=30)$ 
Penicillin-binding protein 3 (PBP3) groups and susceptibility to cephalosporins and carbapenems of Haemophilus influenzae isolates with high-level PBP3-mediated beta-lactam resistance (high-rPBP3), Norway, 2006-2013 (n=30)

\begin{tabular}{|c|c|c|c|c|c|c|c|c|}
\hline \multirow{3}{*}{ Agents } & \multicolumn{4}{|c|}{ Third stage high-rPBP $3^{a}$} & \multicolumn{4}{|c|}{ Second stage high-rPBP $3^{b}$} \\
\hline & \multicolumn{2}{|c|}{ Group III+ $(n=12)$} & \multicolumn{2}{|c|}{ Group III-like+ $(n=4)$} & \multicolumn{2}{|c|}{ Group III $(\mathrm{n}=11)$} & \multicolumn{2}{|c|}{ Group III-like $(n=3)$} \\
\hline & $\begin{array}{l}\mathrm{MIC}_{50} \\
(\mathrm{mg} / \mathrm{L})\end{array}$ & $\begin{array}{l}\mathrm{R} \text { or } \mathrm{I} / \mathrm{R} \\
\mathrm{n}(\%)^{\mathrm{c}}\end{array}$ & $\begin{array}{l}\mathrm{MIC}_{50} \\
(\mathrm{mg} / \mathrm{L})\end{array}$ & $\begin{array}{l}\mathrm{R} \text { or } \mathrm{I} / \mathrm{R} \\
\mathrm{n}(\%)^{\mathrm{c}}\end{array}$ & $\begin{array}{l}\mathrm{MIC}_{50} \\
(\mathrm{mg} / \mathrm{L})\end{array}$ & $\begin{array}{c}\mathrm{R} \text { or } \mathrm{I} / \mathrm{R} \\
\mathrm{n}(\%)^{\mathrm{c}}\end{array}$ & $\begin{array}{l}\mathrm{MIC}_{50} \\
(\mathrm{mg} / \mathrm{L})\end{array}$ & $\begin{array}{l}\mathrm{R} \text { or } \mathrm{I} / \mathrm{R} \\
\mathrm{n}(\%)^{\mathrm{c}}\end{array}$ \\
\hline Cefuroxime & 8 & $1(8) / 11(92)$ & 8 & $\begin{array}{c}\mathrm{O}(0) / \mathrm{t} \\
(100)\end{array}$ & 8 & $\begin{array}{c}0(0) / 11 \\
(100)\end{array}$ & $\geq 16$ & $\begin{array}{c}0(0) / 3 \\
(100)\end{array}$ \\
\hline Cefotaxime & 1 & $12(100)$ & 0.5 & $4(100)$ & 0.25 & $9(82)$ & $\leq 0.12$ & $1(33)$ \\
\hline Ceftriaxone & 0.25 & $10(83)$ & 0.25 & $4(100)$ & 0.06 & $\mathrm{o}(\mathrm{o})$ & 0.06 & $\mathrm{o}(\mathrm{o})$ \\
\hline Cefixime & 0.5 & $12(100)$ & $\geq 2$ & $4(100)$ & 0.25 & $10(91)$ & $\geq 2$ & $3(100)$ \\
\hline Cefepime & 2 & $12(100)$ & 1 & $4(100)$ & 0.5 & $10(91)$ & 0.5 & $2(67)$ \\
\hline Ceftaroline & 0.25 & $12(100)$ & 0.12 & $4(100)$ & 0.06 & $10(91)$ & 0.03 & $\mathrm{o}(\mathrm{o})$ \\
\hline Imipenem & 1 & o (o) & $\leq 0.5$ & o (o) & 1 & o (o) & 1 & o (o) \\
\hline Meropenem & 0.5 & $7(58) / 1(8)^{d}$ & 0.25 & $O(0) / O(0)^{d}$ & 0.25 & $2(18) / 0(0)^{d}$ & 0.12 & $\mathrm{o}(\mathrm{o}) / \mathrm{o}(\mathrm{o})^{\mathrm{d}}$ \\
\hline
\end{tabular}

L389F present. Group III+: S385T, L389F and N526K; group III-like+: S385T, L389F and R517H.

L389F absent. Group III: S385T and N526K; group III-like: S385T and R517H.

According to European Committee on Antimicrobial Susceptibility Testing (EUCAST) clinical breakpoints [6]. The exact breakpoints are presented in Table 2. Isolates categorised as I/R (when an I category is defined) or as R (when only S and R categories exist).

Meningitis breakpoints (0.25/1) used for categorisation. All isolates were susceptible according to general breakpoints (2/2).

\section{Co-resistance}

A total of 11 of 30 strains produced beta-lactamase and all were TEM-1 positive (Table 1). Co-resistance to trimethoprim-sulfamethoxazole (13/30) was frequent. Four of 30 isolates were resistant to chloramphenicol and tetracycline, including the three $\mathrm{CG}_{4}$ isolates and a single isolate belonging to the same clonal complex ( $\mathrm{CC}_{503}$ ) as those forming the $\mathrm{CG}_{4}$. No isolates expressed increased resistance to macrolides or rifampicin (Table 3 ).

Five of 30 isolates had ciprofloxacin MIC $>0.06 \mathrm{mg} / \mathrm{L}$ and possessed amino acid substitutions in the QRDR of GyrA and/or ParC. Three isolates, all belonging to $\mathrm{CG}_{4}$, had substitutions in both proteins and were clinically resistant to ciprofloxacin. Two isolates, both $\mathrm{CG}_{3}$, had only one significant GyrA substitution and quinolone MICs within the susceptible category (Table 4).

\section{Case reports}

The clinical course was evaluated for three patients with well-defined serious infections most likely caused by one of the study isolates (patients and isolates 18 , 27 and 28). Isolate 18 was cultured from the blood of a patient with X-ray confirmed pneumonia, chronic obstructive pulmonary disease (COPD), hypogammaglobulinemia, chronic lymphocytic leukaemia and myasthenia gravis (Table 1). The strain was categorised as susceptible to cefotaxime $(\mathrm{MIC} \leq 0.12 \mathrm{mg} / \mathrm{L}$, Figure 3). The patient responded well to initial parenteral therapy with cefotaxime (three days) followed by ciprofloxacin orally.
Isolates 27 and 28 belonged to $\mathrm{CG}_{5}$ and were beta-lactamase positive and resistant to ampicillin-sulbactam $(\mathrm{MIC}=8 \mathrm{mg} / \mathrm{L})$ and cefotaxime $(\mathrm{MIC}=1 \mathrm{mg} / \mathrm{L})$ according to EUCAST breakpoints [6]. Both were isolated from sputum in patients with X-ray confirmed pneumonia (C-reactive protein $\geq 160 \mathrm{mg} / \mathrm{L}$; norm: $<5 \mathrm{mg} / \mathrm{L}$ ) and significant co-morbidity. Patient 27 (cardiovascular disease) responded to therapy with cefotaxime (dosage $2 \mathrm{~g}$ three times a day) after initial treatment with benzylpenicillin. Patient 28 (disseminated cancer) responded to ciprofloxacin after initial treatment with piperacillin-tazobactam.

\section{Discussion}

We have characterised 30 clinical $H$. influenzae isolates from Norway with high-level $\mathrm{PBP}_{3}$-mediated beta-lactam resistance, including 23 isolates possessing both the $\mathrm{S}_{3} 85 \mathrm{~T}$ and $\mathrm{N}_{526 \mathrm{~K}}$ substitutions. Previously, isolates with this genotype (group III and group III+) have primarily been reported from Japan [14] and Korea [15].

The prevalence of low-rPBP 3 in respiratory $\mathrm{NTHi}$ in Norway has increased gradually since 2001 and was estimated to $15 \%$ in 2007; 113 low-rPBP 3 and three high-rPBP isolates (isolates 4-6 in this study) were reported among 795 surveillance isolates in 2007 [8]. When the prevalence of low-rPBP3 in Japan in the $1990 \mathrm{~s}$ reached approximately the same level as in Norway in 2007, the prevalence of high-rPBP3 isolates increased from zero to $29 \%$ in few years [14]. Similar observations were reported from Korea during the 2000s [15]. NTHi was characterised as an emerging pathogen in a most 
Year-to-year overview of Haemophilus influenzae isolates with high-level penicillin-binding protein 3-mediated beta-lactam resistance (high-rPBP3), Norway, 2006-2013 (n=30)
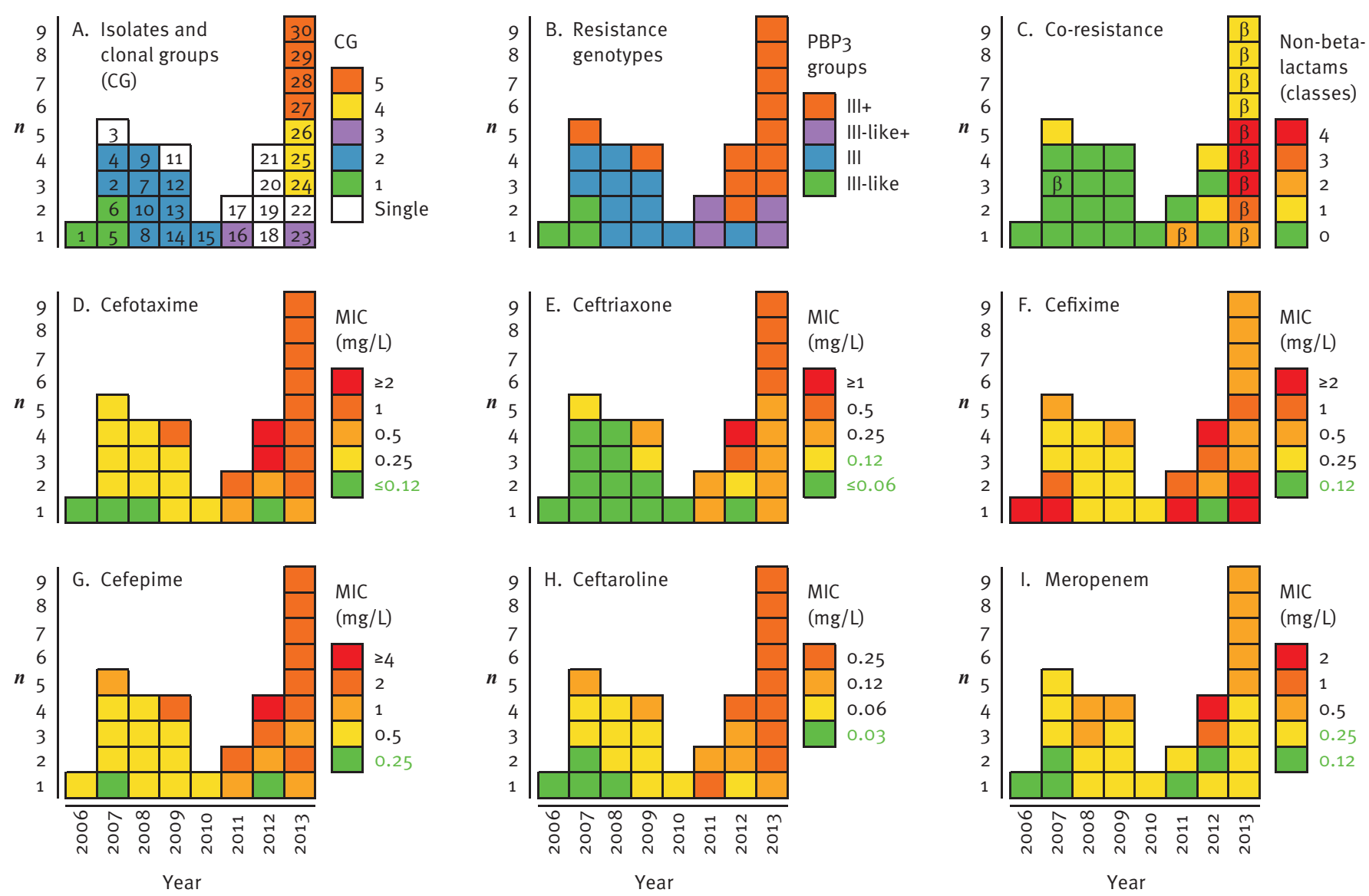

MIC: minimum inhibitory concentration; $\mathrm{PBP}_{3}$ : penicillin-binding protein 3.

Colours indicate the following parameters: A, CGs (identical MLST-fts/ profiles, Table 1); B, PBP3 resistance genotypes (grouped according to substitutions in positions $385,389,517$ and 526 , Table 1 ); $C$, co-resistance to non-beta-lactam antimicrobial agents (number of classes, including low-level resistance to quinolones) and beta-lactamase ( $\beta$ ); D-I, MICs (broth microdilution) to extended-spectrum cephalosporins and meropenem.

Green numbers on the MIC scale indicate values below the European Committee on Antimicrobial Susceptibility Testing (EUCAST) S-breakpoint [6]. Each box indicates the same isolate in all diagrams. Isolates collected the same year (columns) are sorted by PBP3 groups, clonal groups and cefotaxime MIC.

recent review article [1]. Emphasising the emergence and spread of strains with $\mathrm{PBP}_{3}$-mediated resistance, the authors recommended implementation of standardised surveillance protocols and typing methodologies.

We recently suggested MLST-fts/ typing as a tool for molecular surveillance of PBP3-mediated resistance in $H$. influenzae [8]. By this approach, DNA-based resistotyping using the transpeptidase region as an additional allele is combined with MLST allelic profiles. This sequence is readily available if $\mathrm{PBP}_{3}$ resistance genotyping is done by sequencing. In that study [8], MLST clonal complexes corresponded well to PFGE clusters. Addition of the fts/ allele to MLST allelic profiles increased the discriminatory power compared with MLST alone and MLST combined with $\mathrm{PBP}_{3}$ substitution patterns, without compromising consistency with PFGE-based grouping. The observation that $\mathrm{CG}_{2}$ in the present study (and most MLST-fts/ types in the previous study) encompassed related pulsotypes supports the validity of the typing scheme although discriminatory power is inferior to PFGE. Increasing the length of the fts/ fragment could improve resolution but this would most likely be at the cost of reduced sensitivity for clone detection.

So far, the molecular epidemiology of high-rPBP3 strains is poorly described. However, two invasive group III-like isolates recently reported from Spain had MLST allelic profiles (ST155 and ST1118) identical or closely related to $\mathrm{CG}_{1}$ in the present study; the Spanish isolates and $\mathrm{CG}_{1}$ also had identical $\mathrm{PBP}_{3}$ substitution patterns as far as comparison is possible [3]. These observations suggest that $\mathrm{CG}_{1}$ and the two Spanish isolates may be representatives of a virulent high$\mathrm{rPBP}_{3} \mathrm{NTHi}$ clone distributed within Europe. 
TABLE 4

GyrA/ParC amino acid substitutions in Haemophilus influenzae isolates with high-level penicillin-binding protein 3-mediated beta-lactam resistance (high-rPBP3) and raised minimum inhibitory concentrations to quinolones, Norway, $2006-2013(\mathrm{n}=5)^{\mathrm{a}}$

\begin{tabular}{|c|c|c|c|c|c|c|c|}
\hline \multirow{2}{*}{ Isolate ${ }^{b}$} & \multicolumn{2}{|c|}{$\begin{array}{c}\text { MICs (mg/L) and breakpoints } \\
(\mathrm{S} \leq / \mathrm{R}>)^{c}\end{array}$} & \multicolumn{2}{|c|}{ GyrA $^{d}$} & \multicolumn{3}{|c|}{$\operatorname{ParC}^{d}$} \\
\hline & CIP (0.5/0.5) & $\operatorname{LEV}(1 / 1)$ & S84 & D88 & S84 & $\mathrm{N}_{138}$ & $\mathrm{G}_{206}$ \\
\hline 16 & 0.25 & 0.25 & L & - & - & - & $R$ \\
\hline 23 & 0.25 & 0.25 & $\mathrm{~L}$ & - & - & - & $\mathrm{R}$ \\
\hline 24 & 2 & 1 & $\mathrm{~L}$ & $\mathrm{~N}$ & 1 & $\mathrm{~S}$ & - \\
\hline 25 & 2 & 1 & $L$ & $\mathrm{~N}$ & 1 & $\mathrm{~S}$ & - \\
\hline 26 & 2 & 1 & L & $\mathrm{N}$ & I & S & - \\
\hline
\end{tabular}

CIP: ciprofloxacin; LEV: levofloxacin; MIC: minimum inhibitory concentration; -: no substitution.

a The remaining 25 isolates had ciprofloxacin MICs $\leq 0.06 \mathrm{mg} / \mathrm{L}$ and levofloxacin MICs $\leq 0.03 \mathrm{mg} / \mathrm{L}$.

Further characteristics are described in Table 1.

European Committee on Antimicrobial Susceptibility Testing (EUCAST) clinical breakpoints [6].

Amino acid substitutions in GyrA (64-177) and ParC (66-236).

Travel history suggested that one isolate in this study may have been imported from Thailand, although an isolate with identical MLST-fts/ profile was observed in Norway two years earlier (CG3).

In this study, $\mathrm{CG}_{4}$ and $\mathrm{CG}_{5}$ were respectively restricted to one hospital (both hospitals located in Western Norway) and sampled within short time periods. Two of the $\mathrm{CG}_{5}$ isolates were sampled from persons within the same household. Person-to-person transmission of nontypeable $H$. influenzae has been reported previously, including an outbreak with an amoxicillin-resistant strain in a respiratory ward [27] and intrafamilial transmission of $\mathrm{rPBP}_{3}$ strains [28]. These observations highlight the importance of hygiene measures in health institutions to prevent nosocomial spread.

The observation of identically mutated $\mathrm{fts} /$ alleles in unrelated STs suggests horizontal gene transfer (HGT). Similar observations have been reported by us previously [8]. Thus, resistant strains may have developed in Norway by HGT of alleles conferring resistance; these alleles may have been imported at an earlier stage or have evolved locally. The observation of allele mosaicism in $\mathrm{H}$. influenzae [29] supports this interpretation and strongly suggests that allele transfer has occurred via transformation and recombination with the chromosome.

By EUCAST clinical breakpoints [6], all isolates were categorised as resistant to at least one extendedspectrum cephalosporin. Clinically relevant differences in resistance profiles between $\mathrm{PBP}_{3}$ groups were observed. Isolates possessing the third stage substitution $\mathrm{L} 389 \mathrm{~F}$ were generally more resistant to cephalosporins than the remaining isolates, consistent with the results of a previous investigation using sitedirected mutagenesis and transformation to study the impact of $\mathrm{PBP}_{3}$ substitutions on beta-lactam susceptibility [13]. Notably, most $\mathrm{L}_{3} 89 \mathrm{~F}$ positive isolates in the present study were ceftriaxone resistant, while all isolates lacking this substitution were ceftriaxone susceptible. The three-stage classification system used in this study, separating between isolates with and without the $\mathrm{L}_{389} \mathrm{~F}$ substitution, appears to reflect the stepwise development of $\mathrm{PBP}_{3}$-mediated resistance better than systems based on $\mathrm{S}_{3} 85 \mathrm{~T}$ and $\mathrm{R}_{517} \mathrm{H} / \mathrm{N}_{52} 6 \mathrm{~K}$ only.

We evaluated the effectiveness of the antimicrobial therapy for three patients with X-ray confirmed pneumonia associated with study strains. Notably, one patient responded to therapy with high dosage of cefotaxime although the isolate (from sputum) was categorised as resistant $(\mathrm{MIC}=1 \mathrm{mg} / \mathrm{L})$ according to EUCAST clinical breakpoints [6]. This is consistent with current EUCAST non-species related breakpoints for cefotaxime based on a dose of at least $2 \mathrm{~g}$ three times a day $(R>2 \mathrm{mg} / \mathrm{L})$ [6]. The isolate would have been categorised as susceptible to cefotaxime by CLSI breakpoints for $H$. influenzae $(\mathrm{S} \leq 2 \mu \mathrm{g} / \mathrm{mL})[7]$.

The single $\mathrm{ST}_{1287}$ blood isolate from an immunocompromised patient with pneumonia is to our knowledge the first reported invasive group III high-rPBP3 isolate from Europe. This strain generally expressed lower MICs to beta-lactams than noninvasive group III study isolates and was categorised as cefotaxime susceptible $(M I C \leq 0.12 \mathrm{mg} / \mathrm{L})$. The patient responded well to cefotaxime therapy.

Conversely, the single Hif group III+isolate was more resistant than nontypeable isolates with comparable genotypes. These observations suggest that 
strain-associated mechanisms other than $\mathrm{PBP}_{3}$ substitutions may modify resistance levels in $\mathrm{rPBP}_{3}$ isolates. Increased efflux due to mutations in the $a c r R$ gene, encoding a repressor of the AcrAB efflux pump, is associated with high-level macrolide resistance in $H$. influenzae and may increase resistance to ampicillin in $\mathrm{rPBP}_{3}$ strains [30]. The $a c r R$ gene was not sequenced in the present study but all isolates expressed wild-type MICs to macrolides.

Co-resistance was more frequent than expected. The proportions of trimethoprim-sulfamethoxazole resistant and beta-lactamase positive isolates were twice and three times, respectively, the overall reported prevalences in respiratory $H$. influenzae isolates ( $n=$ 677 ) in a Norwegian nationwide surveillance study in 2011 [31]. Similarly, resistance rates for ciprofloxacin, tetracycline and chloramphenicol in this study were $10 \%(3 / 30), 13 \%(4 / 30)$ and $13 \%$ (4/30), respectively, compared with $0.1 \%, 0.4 \%$ and $0.7 \%$ in the 2011 surveillance report.

EUCAST define resistance to ciprofloxacin as an exceptional phenotype in $H$. influenzae and recommend that such isolates are referred to a reference laboratory $[5,6]$. Quinolone resistance in this species is usually due to QRDR substitutions in subunit A of topoisomerase II (GyrA) and subunit A of topoisomerase IV (ParC). Resistance levels depend largely on the number of substitutions in the positions 84 and 88 in both proteins [32]. Isolates with single substitutions are usually low-level resistant but other mechanisms such as the transferable plasmid-mediated acetyl transferase gene $a a c\left(6^{\prime}\right)-l b-c r$ may increase resistance [33,34]. The QRDR substitution patterns and quinolone MICs observed in this study are consistent with previous reports $[26,32]$. The nalidixic acid $30 \mu \mathrm{g}$ disk is superior to the ciprofloxacin $5 \mu \mathrm{g}$ disk for detection of isolates with low-level quinolone resistance and may be used for screening $[6,26]$.

Quinolone resistance in $H$. influenzae is associated with hypermutability [33]. It seems likely that hypermutability also increases the ability to acquire $\mathrm{PBP}_{3}$ substitutions by spontaneous mutations, favouring the development of strains with both resistance mechanisms. To our knowledge, $\mathrm{CG}_{4}$ represent the first ciprofloxacin-resistant high-rPBP3 isolates reported outside Japan, and the first clonal cluster ever with this particular combination of resistance mechanisms.

The $\mathrm{CG}_{4}$ isolates had TEM-1 beta-lactamase and were resistant to chloramphenicol, tetracycline and trimethoprim-sulfamethoxazole. This resistance profile suggests the presence of a conjugative large plasmid [35], now recognised as an integrating and conjugative element (ICE) designated ICEHin1056 [36]. The resistotype of $\mathrm{CG}_{4}$ might thus be the result of two independent factors: hypermutability, leading to $\mathrm{PBP}_{3}$ and QRDR substitutions, and the acquisition of ICEHin1056. Hypermutable $H$. influenzae are prevalent in cystic fibrosis (CF) patients [37]. One $\mathrm{CG}_{4}$ isolate was found in sputum from a CF patient; another isolate was from nasopharynx of a patient with primary ciliary dyskinesia (PCD). High antibiotic pressure in these patient categories may contribute to selection of multidrug resistant strains.

The ST of $\mathrm{CG}_{4}$ (ST159) is associated with increased virulence in chronic obstructive pulmonary disease due to an additional IgA1 protease, encoded by the igaB gene [38].

The emergence of clonally related high-rPBP3 isolates in Norway is a cause for concern. As previously reported from Japan and Korea, a shift from low-level to highlevel $\mathrm{rPBP}_{3}$ might alter the epidemiological situation dramatically in few years $[14,15]$. A similar development in Europe would threaten current recommendations for empiric antimicrobial treatment in severe disease [1]. The situation urges for improved surveillance systems for antimicrobial resistance in $H$. influenzae in Europe.

\section{Acknowledgements}

This work was supported by a grant from Vestfold Hospital Trust. We thank the following for contributing with bacterial isolates and/or clinical data: Haima Mylvaganam (Department of Microbiology, Haukeland University Hospital); Reidar Hjetland and Ingerid Skarstein (Department of Microbiology, Førde Hospital); Truls Leegaard (Department of Microbiology, Akershus University Hospital); Sølvi Noraas and Irene Beate Olsøy (Department of Medical Microbiology, Hospital of Southern Norway Trust); and Pål Arne Jenum (Department of Medical Microbiology, Vestre Viken Hospital Trust). We thank Astrid Lia and Anja Hannisdal (Vestfold Hospital Trust) for excellent technical assistance; Dominique A. Caugant, Elisabeth Fritzsønn and Anne Witsø (Norwegian Institute of Public Health) for performing MLST and capsular serotyping; Andrew Jenkins (Telemark University College, $\mathrm{B} \emptyset)$ for help with analysis of PFGE band patterns; Haima Mylvaganam and Dominique A. Caugant for valuable comments to the manuscript; and the Norwegian Surveillance Programme for Antimicrobial Resistance (NORM) for access to the surveillance database. We also acknowledge use of the Haemophilus influenzae MLST website.

\section{Conflict of interest}

None declared.

\section{Authors' contributions}

DS conceived and coordinated the study; AS and BEK contributed to study design. DS, OBN and MS collected bacterial isolates and clinical information; DS performed or was responsible for testing and characterisation of isolates in collaboration with GK and EM (MIC determination); MS (MLST and capsular serotyping) and ILA (remaining molecular analyses). DS analysed and interpreted data and wrote the first draft. All authors participated in interpretation of results, critically revised the draft for intellectual content and approved the final article. 


\section{References}

1. Van Eldere J, Slack MP, Ladhani S, Cripps AW. Non-typeable Haemophilus influenzae, an under-recognised pathogen. Lancet Infect Dis. 2014;14(12):1281-92. http://dx.doi. org/10.1016/S1473-3099(14)70734-0 PMID:25012226

2. Resman F, Ristovski M, Forsgren A, Kaijser B, Kronvall G, Medstrand $P$, et al. Increase of $\beta$-lactam-resistant invasive Haemophilus influenzae in Sweden, 1997 to 2010. Antimicrob Agents Chemother. 2012;56(8):4408-15. http://dx.doi. org/10.1128/AAC.00415-12 PMID:22687505

3. García-Cobos S, Arroyo M, Pérez-Vázquez M, Aracil B, Lara $\mathrm{N}$, Oteo J, et al. Isolates of $\beta$-lactamase-negative ampicillinresistant Haemophilus influenzae causing invasive infections in Spain remain susceptible to cefotaxime and imipenem. J Antimicrob Chemother. 2014;69(1):111-6. http://dx.doi. org/10.1093/jac/dkt324 PMID:23943391

4. Tristram S, Jacobs MR, Appelbaum PC. Antimicrobial resistance in Haemophilus influenzae. Clin Microbiol Rev. 2007;20(2):368 89. http://dx.doi.org/10.1128/CMR.00040-06 PMID:17428889

5. Leclercq R, Cantón R, Brown DF, Giske CG, Heisig P, MacGowan AP, et al. EUCAST expert rules in antimicrobial susceptibility testing. Clin Microbiol Infect. 2013;19(2):141-60. http://dx.doi. org/10.1111/j.1469-0691.2011.03703.x PMID:22117544

6. The European Committee on Antimicrobial Susceptibility Testing (EUCAST). Breakpoint tables for interpretation of MICS and zone diameters. Version 4.0, 2014. Växjö: EUCAST; 2014. Available from: http://www.eucast.org

7. Clinical and Laboratory Standards Institute (CSLI). Performance standards for antimicrobial susceptibility testing, twentythird informational supplement. Wayne, PA: CLSI; 2013. CLSI document M100-S23

8. Skaare D, Anthonisen IL, Caugant DA, Jenkins A, Steinbakk $M$, Strand L, et al. Multilocus sequence typing and $\mathrm{ftsl}$ sequencing: a powerful tool for surveillance of penicillinbinding protein 3 -mediated beta-lactam resistance in nontypeable Haemophilus influenzae. BMC Microbiol. 2014;14(1):131. http://dx.doi.org/10.1186/1471-2180-14-131 PMID:24884375

9. Ubukata K, Shibasaki Y, Yamamoto K, Chiba N, Hasegawa K, Takeuchi Y, et al. Association of amino acid substitutions in penicillin-binding protein 3 with beta-lactam resistance in beta-lactamase-negative ampicillin-resistant Haemophilus influenzae. Antimicrob Agents Chemother. 2001;45(6):16939. http://dx.doi.org/10.1128/AAC.45.6.1693-1699.2001 PMID:11353613

10. Hotomi M, Fujihara K, Billal DS, Suzuki K, Nishimura T, Baba $S$, et al. Genetic characteristics and clonal dissemination of beta-lactamase-negative ampicillin-resistant Haemophilus influenzae strains isolated from the upper respiratory tract of patients in Japan. Antimicrob Agents Chemother. 2007;51(11):3969-76. http://dx.doi.org/10.1128/AAC.00422-07 PMID:17698631

11. García-Cobos S, Campos J, Lázaro E, Román F, Cercenado E, García-Rey C, et al. Ampicillin-resistant non-beta-lactamaseproducing Haemophilus influenzae in Spain: recent emergence of clonal isolates with increased resistance to cefotaxime and cefixime. Antimicrob Agents Chemother. 2007;51(7):2564-73. http://dx.doi.org/10.1128/AAC.00354-07 PMID:17470649

12. Hasegawa K, Chiba N, Kobayashi R, Murayama SY, Iwata S, Sunakawa K, et al. Rapidly increasing prevalence of betalactamase-nonproducing, ampicillin-resistant Haemophilus influenzae type $b$ in patients with meningitis. Antimicrob Agents Chemother. 2004;48(5):1509-14. http://dx.doi. org/10.1128/AAC.48.5.1509-1514.2004 PMID:15105098

13. Osaki Y, Sanbongi Y, Ishikawa M, Kataoka H, Suzuki T, Maeda $\mathrm{K}$, et al. Genetic approach to study the relationship between penicillin-binding protein 3 mutations and Haemophilus influenzae beta-lactam resistance by using site-directed mutagenesis and gene recombinants. Antimicrob Agents Chemother. 2005;49(7):2834-9. http://dx.doi.org/10.1128/ AAC.49.7.2834-2839.2005 PMID:15980357

14. Ubukata K. Problems associated with high prevalence of multidrug-resistant bacteria in patients with communityacquired infections. J Infect Chemother. 2003;9(4):285-91. http://dx.doi.org/10.1007/s10156-003-0278-Y PMID:14691647

15. Park C, Kim KH, Shin NY, Byun JH, Kwon EY, Lee JW, et al. Genetic diversity of the ftsl gene in $\beta$-lactamase-nonproducing ampicillin-resistant and $\beta$-lactamase-producing amoxicillin-/ clavulanic acid-resistant nasopharyngeal Haemophilus influenzae strains isolated from children in South Korea. Microb Drug Resist. 2013;19(3):224-30. http://dx.doi. org/10.1089/mdr.2012.0116 PMID:23308379

16. Dabernat H, Delmas C. Epidemiology and evolution of antibiotic resistance of Haemophilus influenzae in children 5 years of age or less in France, 2001-2008: a retrospective database analysis. Eur J Clin Microbiol Infect Dis.
2012;31(10):2745-53. http://dx.doi.org/10.1007/s10096-0121623-9 PMID:22538797

17. Skaare D, Allum AG, Anthonisen IL, Jenkins A, Lia A, Strand L, et al. Mutant ftsl genes in the emergence of penicillin-binding protein-mediated beta-lactam resistance in Haemophilus influenzae in Norway. Clin Microbiol Infect. 2010;16(8):111724. http://dx.doi.org/10.1111/j.1469-0691.2009.03052.x PMID:19737286

18. Barbosa AR, Giufrè M, Cerquetti M, Bajanca-Lavado MP. Polymorphism in ftsl gene and beta-lactam susceptibility in Portuguese Haemophilus influenzae strains: clonal dissemination of beta-lactamase-positive isolates with decreased susceptibility to amoxicillin/clavulanic acid. I Antimicrob Chemother. 2011;66(4):788-96. http://dx.doi. org/10.1093/jac/dkq533 PMID:21393206

19. Shuel M, Hoang L, Law DKS, Tsang R. Invasive Haemophilus influenzae in British Columbia: non-Hib and non-typeable strains causing disease in children and adults. Int J Infect Dis. 2011;15(3):e167-73. http://dx.doi.org/10.1016/j.ijid.2010.10.005 PMID:21134777

20. Kilian M. A taxonomic study of the genus Haemophilus, with the proposal of a new species. I Gen Microbiol. 1976;93(1):962. http://dx.doi.org/10.1099/00221287-93-1-9 PMID:772168

21. Falla TJ, Crook DW, Brophy LN, Maskell D, Kroll JS, Moxon ER. PCR for capsular typing of Haemophilus influenzae. J Clin Microbiol. 1994;32(10):2382-6. PMID:7814470

22. Meats E, Feil EJ, Stringer S, Cody AJ, Goldstein R, Kroll JS, et al. Characterization of encapsulated and noncapsulated Haemophilus influenzae and determination of phylogenetic relationships by multilocus sequence typing. J Clin Microbiol. 2003;41(4):1623-36. http://dx.doi.org/10.1128/JCM.41.4.16231636.2003 PMID:12682154

23. Tenover FC, Arbeit RD, Goering RV, Mickelsen PA, Murray BE, Persing $\mathrm{DH}$, et al. Interpreting chromosomal DNA restriction patterns produced by pulsed-field gel electrophoresis: criteria for bacterial strain typing. J Clin Microbiol. 1995;33(9):2233-9. PMID:7494007

24. Livermore DM, Brown DFJ. Detection of beta-lactamasemediated resistance. J Antimicrob Chemother. 2001;48(Suppl 1):59-64. http://dx.doi.org/10.1093/jac/48.suppl_1.59 PMID:11420337

25. Clinical and Laboratory Standards Institute (CSLI). Mo7-A9. Methods for dilution antimicrobial susceptibility tests for bacteria that grow aerobically; approved standard - ninth edition. Wayne, PA: CSLI;,2012.

26. Pérez-Vázquez M, Román F, Aracil B, Cantón R, Campos J. Laboratory detection of Haemophilus influenzae with decreased susceptibility to nalidixic acid, ciprofloxacin, levofloxacin, and moxifloxacin due to GyrA and ParC mutations. J Clin Microbiol. 2004;42(3):1185-91. http://dx.doi. org/10.1128/JCM.42.3.1185-1191.2004 PMID:15004073

27. Hekker TA, van der Schee AC, Kempers J, Namavar F, van Alphen L. A nosocomial outbreak of amoxycillin-resistant non-typable Haemophilus influenzae in a respiratory ward. Hosp Infect. 1991;19(1):25-31. http://dx.doi.org/10.1016/01956701(91)90125-R PMID:1684594

28. Watanabe H, Hoshino K, Sugita R, Asoh N, Watanabe K, Oishi $\mathrm{K}$, et al. Possible high rate of transmission of nontypeable Haemophilus influenzae, including beta-lactamase-negative ampicillin-resistant strains, between children and their parents. J Clin Microbiol. 2004;42(1):362-5. http://dx.doi. org/10.1128/JCM.42.1.362-365.2004 PMID:14715779

29. Witherden EA, Bajanca-Lavado MP, Tristram SG, Nunes A. Role of inter-species recombination of the ftsl gene in the dissemination of altered penicillin-binding-protein-3-mediated resistance in Haemophilus influenzae and Haemophilus haemolyticus. J Antimicrob Chemother. 2014;69(6):1501-9. http://dx.doi.org/10.1093/jac/dku022 PMID:24562614

30. Kaczmarek FS, Gootz TD, Dib-Hajj F, Shang W, Hallowell $S$, Cronan M. Genetic and molecular characterization of beta-lactamase-negative ampicillin-resistant Haemophilus influenzae with unusually high resistance to ampicillin. Antimicrob Agents Chemother. 2004;48(5):1630-9. http:// dx.doi.org/10.1128/AAC.48.5.1630-1639.2004 PMID:15105114

31. NORM/NORM-VET. Usage of Antimicrobial Agents and Occurrence of Antimicrobial Resistance in Norway. Troms $\varnothing$ / Oslo, Norway. NORM/NORM-VET; 2011. Available from: http://www.unn.no/getfile.php/UNN\%2oINTER/Fagfolk/www. antibiotikaresistens.no/NORM 2012/NORM\%20NORM-VET\%20 2011.pdf

32. Georgiou M, Muñoz R, Román F, Cantón R, Gómez-Lus $R$, Campos ], et al. Ciprofloxacin-resistant Haemophilus influenzae strains possess mutations in analogous positions of GyrA and ParC. Antimicrob Agents Chemother. 1996;40(7):17414. PMID:8807076 
33. Pérez-Vázquez M, Román F, García-Cobos S, Campos J. Fluoroquinolone resistance in Haemophilus influenzae is associated with hypermutability. Antimicrob Agents Chemother. 2007;51(4):1566-9. http://dx.doi.org/10.1128/ AAC.01437-06 PMID:17283196

34. Pfeifer Y, Meisinger I, Brechtel K, Gröbner S. Emergence of a multidrug-resistant Haemophilus influenzae strain causing chronic pneumonia in a patient with common variable immunodeficiency. Microb Drug Resist. 2013;19(1):1-5. http:// dx.doi.org/10.1089/mdr.2012.0060 PMID:23095085

35. Leaves NI, Dimopoulou I, Hayes I, Kerridge S, Falla T, Secka O, et al. Epidemiological studies of large resistance plasmids in Haemophilus. J Antimicrob Chemother. 2000;45(5):599-604. http://dx.doi.org/10.1093/jac/45.5.599 PMID:10797080

36. Mohd-Zain Z, Turner SL, Cerdeño-Tárraga AM, Lilley AK, Inzana TJ, Duncan AJ, et al. Transferable antibiotic resistance elements in Haemophilus influenzae share a common evolutionary origin with a diverse family of syntenic genomic islands. J Bacteriol. 2004;186(23):8114-22. http://dx.doi.org/10.1128/ JB.186.23.8114-8122.2004 PMID:15547285

37. Watson ME Jr, Burns JL, Smith AL. Hypermutable Haemophilus influenzae with mutations in mutS are found in cystic fibrosis sputum. Microbiology. 2004;150(Pt 9):2947-58. http://dx.doi. org/10.1099/mic.0.27230-o PMID:15347753

38. Murphy TF, Lesse AJ, Kirkham C, Zhong H, Sethi S, Munson RS Jr. A clonal group of nontypeable Haemophilus influenzae with two IgA proteases is adapted to infection in chronic obstructive pulmonary disease. PLoS ONE. 2011;6(10):e25923. http:// dx.doi.org/10.1371/journal.pone.0025923 PMID:21998721 